\title{
Dawn of cardiac surgery in Japan
}

\author{
Akira Furuse, MD
}

(C) The Japanese Association for Thoracic Surgery 2007

\section{Erratum to: Gen Thorac Cardiovasc Surg DOI 10.1007/s11748-006-0064-8}

There was an error in the "Open heart surgery under hypothermia" section of the above-mentioned article. On the fourth line in the right column, the date of the surgery should be January 17, 1955.

The online version of the original article can be found at http://dx.doi.org/10.1007/s11748-006-0064-8
A. Furuse
JR Tokyo General Hospital, Shibuya-ku, Tokyo, Japan 